\title{
Polimorfismos FecXR, FecG y FecGH en el ovino de pelo colombiano
}

Hernández, D. ${ }^{1}$; Montes, D. ${ }^{1}$; Otero, R. ${ }^{\text {; }}$ Prieto, E. ${ }^{1}$; Espitia, A. ${ }^{1}$ y Buelvas, M. ${ }^{2}$

'Facultad de Ciencias Agropecuarias. Universidad de Sucre. Sincelejo. Colombia.

${ }^{2}$ Asovicor. Montería, Córdoba. Colombia.

Palabras Clave adicionales

Gen BMP15.

Gen GDF9.

Prolificidad.

Recurso zoogenético.

\section{RESUMEN}

El ovino de pelo criollo (OPC) es un recurso zoogenético de gran importancia para Colombia, no existen programas de mejoramiento genético en el OPC, por lo que los cruzamientos absorbentes son los más comunes con el fin de aumentar la prolificidad. No se han estudiado los genes con efecto mayor sobre la prolificidad en el OPC, por lo que el objetivo de esta investigación fue caracterizar el polimorfismo genético en el locus Fec $X^{R}$ del gen BMP15 y los loci FecG ${ }^{H}$ y FecG' del gen GDF9. Para ello, se genotiparon 150 ovinos por PCR directa para el locus FecX ${ }^{R}$ y por PCR-RFLP para los loci FecG ${ }^{H}$ y FecG'. Se estimaron las frecuencias genotípicas y alélicas, se calculó la heterocigocidad observada $(\mathrm{Ho})$ y esperada $(\mathrm{He})$, los valores de $\mathrm{F}$, el equilibrio de Hardy-Weinberg (EHW) y un análisis de varianza molecular (AMOVA). Los loci Fec $X^{R}$ y FecG $G^{H}$ fueron monomórficos, solo se encontró el genotipo y alelo silvestre en cada uno. El locus FecG' presentó frecuencias de 0,76 y 0,24 en los genotipos $G G$ y $G A$, respectivamente. No se encontró el genotipo $A A$. Las frecuencias alélicas fueron $G=0,89$ y $A=0,11$. Las frecuencias aquí presentadas concuerdan con varios reportes en razas al rededor del mundo. La Ho fue mayor $(0,226 \pm 0,15)$ que la $\mathrm{He}(0,189 \pm 0,12)$, con exceso de heterocigotos dentro $\left(F_{1 S}=-0,127, p>0,05\right)$ y entre los rebaños $\left(F_{I T}=-0,119, p>0,05\right)$. La variación entre rebaños fue baja $(1 \%)$ y el $F_{S T}$ fue de $0,008(p>0,05)$. La población se encontró en EHW. Este es el primer reporte de estos polimorfismos en el OPC que podrían ser utilizados en esquemas de selección para mejorar la prolificidad en esta raza.

\section{FecX' ${ }^{\mathrm{R}}$ FecG' and FecG ${ }^{\mathrm{H}}$ polymorphisms in Colombian hair sheep}

\section{ADDITIONAL KEYWORDS}

BMP15 gene.

GDF9 gene.

Prolificity.

Animal genetic resource.

\section{INFORMATION}

\section{Cronología del artículo.}

Recibido/Received: 11.12 .2017

Aceptado/Accepted: 30.01 .2018

On-line: 07.04 .2019

Correspondencia a los autores/Contact e-mail:

darwin.hernandez@unisucre.edu.co

\section{SUMMARY}

The Creole hair sheep (OPC) is an animal genetic resource of great importance for Colombia, there are no breeding programs in the OPC, so the absorbent crossings are the most common in order to increase prolificacy. The genes with greater effect on the prolificity in the OPC have not been studied, so the objective of this investigation was to characterize the genetic polymorphism in the FecX locus of the BMP15 gene and the $\mathrm{FecG}^{\mathrm{H}}$ and FecG' loci of the GDF9 gene. For this, 150 sheep were genotyped by direct PCR for the FecXR locus and by PCR-RFLP for the FecG $\mathrm{G}^{\mathrm{H}}$ and FecGl loci. The genotypic and allelic frequencies were estimated, the heterozygosity observed $(\mathrm{Ho})$ and expected $(\mathrm{He})$, the $\mathrm{F}$ values, the HardyWeinberg equilibrium (HWE) and a molecular variance analysis (AMOVA) were calculated. The Fec $X^{R}$ and $F e c G^{H}$ loci were monomorphic, only the genotype and allele wild were found in each one. The FecG' locus presented frequencies of 0,76 and 0,24 in the genotypes GG and $G A$, respectively. The $A A$ genotype was not found. The allele frequencies were $G=$ 0,89 and $A=0,11$. The frequencies presented here agree with several reports on races around the world. The Ho was higher $(0,266 \pm 0,15)$ than the $\mathrm{He}(0,189 \pm 0,12)$, with excess of heterozygotes within $\left(F_{1 S}=-0,127, p>0,05\right)$ and between herds $\left(F_{I T}=-0,119, p>0,05\right)$. The variation between herds was low $(1 \%)$ and the $F_{S T}$ was $0,008(p>0,05)$. The population was found in EHW. This is the first report of these polymorphisms in the OPC that could be used in selection schemes to improve prolificacy in this breed.

\section{INTRODUCCIÓN}

Los ovinos que se trajeron durante la conquista de América, constituyeron la base racial de lo que hoy día se conoce como el ovino criollo (Vivas 2013, p. 10). En Colombia se distinguen dos tipos de ovinos, el criollo de lana (CRL) y el ovino de pelo colombiano (OPC), tiene un censo de 1.423 .466 animales, donde cuatro departamentos tienen el 74\% de la población (Ocampo et al. 2017, p. 118). En Colombia la producción ovina se realiza en sistemas tradicionales y familiares, con bajos requerimiento de insumos, los cuales están general- 
mente relacionados con sistemas mixtos de produción junto con bovinos y caprinos (Castellanos et al. 2010, p. 22).

El ovino CRL y el OPC presentan características adaptativas importantes al clima tropical, tales como la tolerancia al calor, ectoparásitos y la capacidad de consumir pasturas de escaso valor nutricional (Carrillo-Gonzalez \& Hernández 2016, p. 198). A pesar de lo anterior, dichos sistemas de producción tienen problemas de nutrición, reproducción, sanidad, desordenados planes de mejora genética, instalaciones inadecuadas y escasa visión empresarial, que se refleja, entre otras, en una baja eficiencia productiva (Cuellar et al. 2015, p. 460).

La eficiencia reproductiva del rebaño, puede ser medida por medio de varios parámetros, entre ellos, la fertilidad (cantidad de ovejas preñadas o paridas sobre el número de ovejas encarneradas), la prolificidad (número de corderos nacidos sobre el total de ovejas paridas) y la supervivencia del cordero (número de corderos destetados sobre número de corderos nacidos) (Buratovich 2010, p. 2). De estos tres parámetros la prolificidad es uno de los más estudiados. El aumento de la prolificidad se da principalmente por un aumento en la tasa de ovulación, el número de óvulos fertilizados y la sobrevivencia embrionaria, que ocasiona un aumento del número de partos dobles y triples (Alabart et al. 2016, p 453).

La tasa ovulatoria está determinada por efectos genéticos y no genéticos. En los primeros, la acción de genes únicos con efecto mayor son llamados genes de fecundidad (Fec) (Davis 2005, p. S2). Uno de estos está localizado en el cromosoma X y se denomina FecX, el gen que ocupa este locus es el de la proteína morfogénica ósea 15 (Bone Morphogenetic Protein 15 - BMP15) del cual se conocen seis fenotipos llamados: Inverdale $\left(\mathrm{Fec}^{\mathrm{I}}\right)$, Hanna $\left(\mathrm{Fec} \mathrm{X}^{\mathrm{H}}\right)$, Belclare $\left(\mathrm{Fec} \mathrm{X}^{\mathrm{B}}\right)$, Galway $(\mathrm{Fe}-$ $\left.\mathrm{cX}^{\mathrm{G}}\right)$, Lacaune $\left(\mathrm{Fec} \mathrm{X}^{\mathrm{L}}\right)$ y Roa $\left(\mathrm{Fec} \mathrm{X}^{\mathrm{R}}\right)$ (Lahoz et al. 2014, pp 3523). Alelo $F e c X^{R}$, fue descrito por primera vez en la raza ovina aragonesa "Rasa Oviaragón", la cual es una raza autóctona española que produce un cordero de calidad llamado Ternasco de Aragón. La variante Fec $X^{\mathrm{R}}$ consiste en una deleción de 17 pares de bases (c. 525_541delTGGGTCCAGAAAAGCCC), que lleva a una alteración en la secuencia de aminoácidos, introduciendo un codón de paro prematuro, por lo que no se sintetiza el péptido maduro (Monteagudo et al. 2009, p. 142). Según Lahoz et al. (2014, p 3524) la variante génica $F e c X^{R}$ da lugar a un incremento de la tasa de ovulación de $+0,63$ ovulaciones por oveja en animales heterocigotos, que ocasiona un incremento de la prolificidad de 0,35 corderos por parto $\left(1,69+/ \mathrm{Fec}^{\mathrm{R}}\right.$ vs. $1,34+/+, \mathrm{p}<0,0001)$, además, las ovejas homocigotas $\mathrm{Fec}^{\mathrm{R}} / \mathrm{Fec}^{\mathrm{R}}$ son estériles.

Otro gen relacionado es el GDF9 (Growth Differentiation Factor 9) que se encuentra en el cromosoma 5, este locus es también llamado FecG. Este gen solo se expresa en el ovario y es requerido para la foliculogénesis, ya que, promueve el desarrollo del folículo primordial, estimula la proliferación de células de la granulosa, impulsa la transición celular de las fases G0 / G1 a S y G2 / M, a través de un aumento de la expresión de CCND1 y CCNE1, y la fosforilación de RB1, regula la expresión de STAR y la liberación de progesterona dependiente de cAMP en células granulosas y tecales, atenúa los efectos supresores de la activina A sobre la expresión de STAR y la producción de progesterona mediante el aumento de la expresión de la inhibina B y suprime la producción de FST y FSTL3 en células granulosas (Liao et al. 2004, p. 17392). Del gen GDF9 se conocen principalmente tres fenotipos: High fertility $\left(\mathrm{FecG}^{\mathrm{H}}\right)$, Embrapa $\left(\mathrm{FecG}^{\mathrm{E}}\right)$ y $\mathrm{G} 1\left(\mathrm{FecG}^{\mathrm{I}}\right)$ (Moradband et al. 2011, p. 1180).

El polimorfismo $\mathrm{FecG}^{\mathrm{H}}$ es una mutación de C1184T que resulta en el cambio de una Serina por una Fenilalanina en la posición 77 del péptido maduro, este polimorfismo cambia el modo de interacción del receptor ligador de dominio tipo 1 de la proteína, por lo que el desarrollo ovárico desde la etapa pre-antral hasta la de crecimiento folicular es diferente en todos los genotipos (Luna et al. 2014, p. 116). De este modo, Los animales homocigotos TT para este alelo no ovulan y son infértiles, mientras que en los animales heterocigotos (CT) la tasa de ovulación promedio es de +2 comparados con las de tipo silvestre (CC), por lo que la ventaja en fertilidad, prolificidad y tasa ovulatoria es sólo para los heterocigotos (Hanrahan et al. 2004, p. 902; Barzegari et al. 2010, p. 667). El polimorfismo FecG $^{\mathrm{I}}$ (G/A) provoca el cambio de una Arginina por una Histidina en la posición 87 del exón 1, la naturaleza química de estos aminoácidos hace suponer que no afecte la actividad de la proteína madura (Moradband et al. 2011, p. 1179).

En el OPC no se han estudiado los genes con efecto mayor sobre la prolificidad. Por tanto, el objetivo de esta investigación fue caracterizar el polimorfismo genético en el locus Fec $\mathrm{X}^{\mathrm{R}}$ del gen BMP15 y los loci $\mathrm{FecG}^{\mathrm{H}}$ y FecG del gen GDF9.

\section{MATERIAL Y MÉTODOS}

\section{ANIMALES Y EXTRACCIÓN DE ADN}

Se colectó sangre periférica mediante punción de la vena yugular usando el sistema Vacutainer ${ }^{\circledR}$ en tubos con anticoagulante (EDTA 7,2 mg) a 150 hembras de la raza ovina de pelo colombiano (OPC) adultas con historial de partos y clínicamente sanos, provenientes de nueve rebaños, ubicadas en el departamento de Córdoba, Colombia. El ADN se extrajo usando el estuche comercial QIAamp® DNA Mini Kit de QIAGEN. Se evaluó la cantidad y calidad del ADN extraído mediante un análisis de absorbancia usando un espectrofotómetro NanoDrop 2000 ${ }^{\mathrm{TM}}$ (Thermo Fisher Scientific). El ADN fue diluido a $10 \mathrm{ng} / \mu \mathrm{l}$.

AmplificaCión y GenOtipificación de lOS lOCI FeCX', FECG $^{\mathrm{H}}$ Y FECG

El locus Fec $X^{\mathrm{R}}$ fue genotipado mediante una PCR directa pues el polimorfismo consiste en una delección de 17 nucleótidos (Martínez-Royo et al, 2008, p. 2). Los loci $\mathrm{FecG}^{\mathrm{H}}$ y FecG $\mathrm{F}^{\mathrm{I}}$ fueron genotipados por PCR-RFLP (Paz et al. 2014, p. 329). En la tabla I se presentan las secuencias de los cebadores y las enzimas de restricción utilizadas. 
Tabla I. Cebadores, tamaño de amplificados y enzimas de restricción utilizadas en cada locus estudiado (Primers, size of amplified and restriction enzymes used in each locus studied).

\begin{tabular}{|c|c|c|c|c|}
\hline Gen & Locus & Cebadores & Tamaño de amplificado & Enzima de restricción \\
\hline \multirow{2}{*}{ BPM151 } & \multirow{2}{*}{ FecX } & F 5'-CTCTGAGACCAAACCGGGTA-3' & \multirow{2}{*}{$160 \mathrm{pb}$} & \multirow{2}{*}{----} \\
\hline & & R 5'-TTTGAGGAGCCTCTT CCTGA-3' & & \\
\hline \multirow{4}{*}{ GDF9² } & \multirow{2}{*}{ FecG' } & F 5'-GAAGACTGGTATGGGGAAATG-3' & \multirow{2}{*}{$463 \mathrm{pb}$} & \multirow{2}{*}{ Hhal } \\
\hline & & R 5'-CCAATCTGCTCCTACACACCT-3' & & \\
\hline & \multirow{2}{*}{$\mathrm{FecG}^{\mathrm{H}}$} & F 5'-CTTTAGTCAGCTGAAGTGGGACAAC-3' & \multirow{2}{*}{$139 \mathrm{pb}$} & \multirow{2}{*}{ Ddel } \\
\hline & & R 5'-ATGGATGATGTTCTGCACCATGGTGTGAACCTGA-3' & & \\
\hline
\end{tabular}

${ }^{1}$ Martínez-Royo et al, 2008, p. 2. ${ }^{2}$ Paz et al. 2014, p. 329

Todas las reacciones de PCR fueron llevadas a cabo en un volumen final de $12,5 \mu$ que contenían $20 \mathrm{ng}$ de $\mathrm{ADN}, 250 \mathrm{nM}$ de cada cebador y $1 \mathrm{X}$ del súper mix MangoMix ${ }^{\mathrm{TM}}$ (Bioline@). El perfil de amplificación de los tres polimorfismos incluyó una desnaturalización inicial de $95^{\circ} \mathrm{C}$ por 5 minutos, seguidos de 30 ciclos de desnaturalización a $95^{\circ} \mathrm{C}$ por 30 segundos, hibridación a $60^{\circ} \mathrm{C}$ por 30 segundos, extensión a $72^{\circ} \mathrm{C}$ por 30 segundos, y una extensión final de $72^{\circ} \mathrm{C}$ por 5 minutos. Las reacciones fueron realizadas en un termociclador MasterCycler Nexus Gradient de Eppendorf®. El RFLP se realizó en un volumen final de $12,5 \mu$ que contenían $3 \mu \mathrm{l}$ de producto de PCR, $1 \mathrm{X}$ del Buffer Tango y $10 \mathrm{U}$ de cada, el mix de digestión se incubó por 3 horas a $37^{\circ} \mathrm{C}$ y 20 minutos a $80^{\circ} \mathrm{C}$.

El producto de PCR del locus Fec $X^{\mathrm{R}}$ y los productos de restricción de los loci $\mathrm{FecG}^{\mathrm{I}}$ y FecG $\mathrm{F}^{\mathrm{H}}$ fueron mezclados en proporción 1:4 con GelRed (Biotium, Inc. EUA) y sometidos a electroforesis vertical en geles de poliacrilamida 37:1 (acrilamida: bisacrilamida) al 12\% a 160 voltios durante 45 minutos y observados bajo luz ultra violeta. Los genotipos fueron asignados según lo determinado por Paz et al. (2014, p. 328) y MartínezRoyo et al. (2008, p. 2).

\section{ANÁLISIS DE DATOS}

Se calcularon las frecuencias genotípicas, las frecuencias alélicas, la heterocigocidad observada $(\mathrm{Ho})$ y esperada (He), el índice F y las desviaciones del equilibrio de Hardy-Weinberg (EHW). Se realizó un análisis de varianza molecular (AMOVA) y se calcularon los F de Wright, para esto se consideró el rebaño de origen de cada animal. Todos los análisis se realizaron con el programa GENALEX versión 6.5 (Peakall \& Smouse 2012, pp. 2537-39).

\section{RESULTADOS}

Las frecuencias de los genotipos y los alelos para cada loci se muestran en la tabla II. Los loci Fec $X^{\mathrm{R}}$ y $\mathrm{FecG}^{\mathrm{H}}$ fueron monomórficos, solo se encontraron los genotipos silvestres por lo que no se halló los alelos mutados de cada polimorfismo $\left(\mathrm{Fec} \mathrm{X}^{\mathrm{R}}\right.$ y $\mathrm{T}$, respectivamente). El locus FecG fue polimórfico, con mayor frecuencia del genotipo silvestre (GG) y con la ausencia del genotipo mutado (AA). La frecuencia del alelo A en este locus fue menor que la del otro alelo.
Los valores de $\mathrm{Ho}$, $\mathrm{He}$, F y EHW, solo se estimaron para el locus FecG $\mathrm{G}^{\mathrm{I}}$ en razón a que los loci $\mathrm{Fec}^{\mathrm{R}}$ y Fe$\mathrm{cG}^{\mathrm{H}}$ fueron monomórficos. Se encontró en el locus FecG $\mathrm{G}^{\mathrm{I}}$ un valor de Ho de 0,226 $\pm 0,15$ y de 0,189 $\pm 0,12$ de He, que tuvo como consecuencia un valor de F de -0,194. El locus FecGI no presentó desviaciones significativas $(p>0,05)$ de EHW.

El AMOVA separó la variación total en dos fuentes, entre rebaños y dentro de individuos, el porcentaje de variación en esta última fuente fue del $99 \%$. El valor de $\mathrm{F}_{\mathrm{ST}}$ calculado a partir de este análisis de varianza fue de 0,008 ( $p>0,05)$, los valores de $\mathrm{F}_{\text {IS }}$ y $\mathrm{F}_{\text {IT }}$ fueron $-0,127$ y $-0,119$, respectivamente, sin diferencias estadísticas significativas.

\section{DISCUSIÓN}

Este es el primer reporte de evaluación del polimorfismo en genes candidatos a prolificidad en el OPC.

Alelo Fec $X^{R}$ aumenta de la tasa de ovulación en $+0,63$ ovulos/oveja en animales heterocigotos, que ocasiona un incremento de la prolificidad de 0,35 (Lahoz et al. 2014, p 3524). En la presente investigación la mutación Fec $X^{\mathrm{R}}$ no se encontró, además, no existen reportes de literatura de la evaluación de este polimorfismo en otras razas alrededor del mundo.

En este estudio se encontró una frecuencia del alelo mutado FecG $\mathrm{I}^{\mathrm{I}}$ de $11 \%$ (Alelo A). El único reporte de análisis de este polimorfismo en razas criollas lo presenta Paz et al. (2014, p. 330) en la raza Araucana de Chile, con frecuencia de 22\%. En la raza Corriedale la frecuencia reportada es de 27\% (Vera et al. 2014, p. 18). Mayores frecuencias de este alelo se reportan en las razas Afshari, Baluchi, Makui y Merhaban por Javanmard et al. (2011, p.160). Mientras que, frecuencias más bajas son presentadas por Liandris et al. $(2012$, p. 18) en la raza Karagouniki y por Ghaderi et al. (2010, p.8022) en las razas Arabic y Kordi.

Cabe destacar que en esta investigación que no fue posible encontrar individuos con genotipo AA. De otro lado, este genotipo si se encontró en las razas Sangsari (1\%) (Kasiriyan et al. 2010, p. 33), Chios (10\%) (Liandris et al. 2012, p.18) y Baluchi con frecuencias entre 6 y 10\% (Javanmard et al. 2011, p.160; Liandris et al. 2012, p.18). En todos los reportes antes citados, la frecuencia del genotipo silvestre fue mayor que la 
Tabla II. Frecuencias genotípicas y alélicas para los tres loci estudiados en el OPC (Genotypic and allelic frequencies for the three loci studied in the OPC).

\begin{tabular}{|c|c|c|c|c|c|}
\hline \multirow{3}{*}{$\begin{array}{l}\text { Locus } \\
\text { FecX }^{\mathrm{R}}\end{array}$} & \multicolumn{3}{|c|}{ Frecuencias genotípicas } & \multicolumn{2}{|c|}{ Frecuencias alélicas } \\
\hline & $+/+$ & $+/ F_{e c X^{R}}$ & $\mathrm{FecX}^{\mathrm{R}} / \mathrm{Fec}^{\mathrm{R}}$ & + & $\mathrm{FecX}^{\mathrm{R}}$ \\
\hline & 1,00 & 0,00 & 0,00 & 1,00 & 0,00 \\
\hline \multirow{2}{*}{ FecG' } & GG & GA & AA & G & $A$ \\
\hline & 0,78 & 0,22 & 0,00 & 0,89 & 0,11 \\
\hline \multirow{2}{*}{$\mathrm{FecG}^{\mathrm{H}}$} & $\mathrm{CC}$ & $\mathrm{CT}$ & TT & C & $\mathrm{T}$ \\
\hline & 1,00 & 0,00 & 0,00 & 1,00 & 0,00 \\
\hline
\end{tabular}

del genotipo heterocigoto (GA), lo que concuerda con nuestros resultados.

Respecto al efecto del genotipo en el locus FecG sobre la prolificidad, varios autores presentan datos diferentes. Hanrahan et al. (2004, pp. 900-9) no encontraron efecto de ningún genotipo. Paz et al. (2014, p. 328) señalan que los animales con genotipo AA resultan ser estériles y por tanto que serían eliminados rápidamente de los rebaños, en esta investigación solo se tomaron muestras de ADN de individuos adultos con historial de partos lo que podría explicar porque no se encontró este genotipo en la muestra poblacional estudiada. Liandris et al. (2012, p.19) reportaron un aumento de la prolificidad en individuos de raza Chios que tienen el genotipo AA (2,25 corderos/hembra/ parto) respecto a los otros genotipos GA y GG con prolificidad promedio de 1,45 y 1,59, respectivamente. Por último, Moradband et al. (2011, p.1182) en la raza india Baluchi, reportaron que el genotipo más prolífico es el heterocigoto GA $(1,38 \pm 0,05)$ respecto a los otros dos genotipos GG $(1,23 \pm 0.03)$ y AA $(1,03 \pm 0,05)$.

La ausencia del polimorfismo $\mathrm{FecG}^{\mathrm{H}}$ concuerdan con los resultados presentados por Paz et al. (2014, p. 330) en la raza Araucana de Chile, por Ghaderi et al. (2010, p.8022) en las razas Arabic y Kordi y por Vacca et al. (2010, p. 69) en las razas del norte de Âfrica Barbarine, Queue Fine de L'Ouest, Noire de Thibar, SiciloSarde y D'man. De otro lado, Liandris et al. (2012, p.18) en la raza Chios se reportan frecuencias de 65,11 y $24 \%$ para los genotipos CC, CT y TT, respectivamente y en la raza Karagouniki de 76, 24 y $0 \%$ para los genotipos CC, CT y TT, respectivamente. Al igual que los últimos autores, la mutación $\mathrm{FecG}^{\mathrm{H}}$ fue reportada también en las razas Belclare y Cambridge (Hanrahan et al. 2004, p. 902; Ghaffari et al. 2009, p. 357).

Según nuestros datos, en el OPC, los alelos FecX ${ }^{R}$ y FecG ${ }^{\mathrm{H}}$ no se encontraron en la muestra poblacional analizada. La no presencia de estos polimorfismos puede ser atribuida a cuatro razones, la primera es a efectos de deriva genética que imposibilitaron muestrear un animal con alguna de las mutaciones, la segunda es que dichos polimorfismos estén fijos en las razas en las cuales fueron reportados $\mathrm{Fec}^{\mathrm{R}}$ en la raza Aragonesa (Monteagudo et al. 2009, pp. 139-46) y $\mathrm{FecG}^{\mathrm{H}}$ en las razas Belclare y Cambridge (Hanrahan et al. 2004, p. 902), la tercera es que estas razas no hayan participado en la formación del OPC teniendo en cuenta que parte del origen de esta última es Ibérico y la cuarta es que debido a la ausencia de planes de manejo reproductivo y de mejoramiento genético animal en el OPC la mutación se perdiera ya que según Ocampo et al. (2017, p. 118) el mejoramiento genético en el OPC se basan en cruzamientos de tipo absorbente con razas importadas para aprovechar el vigor híbrido.

La heterosigocidad esperada (He) es considerada como uno de los mejores estimadores de variabilidad genética (Kim et al. 2002, p. 203). En el presente estudio la heterocigocidad no se calculó en los loci $\mathrm{Fec}^{\mathrm{R}} \mathrm{y}$ $\mathrm{FecG}^{\mathrm{H}}$ y tuvo un valor promedio de 0,189 para el locus FecG ${ }^{\mathrm{I}}$ con valores negativos de $\mathrm{F}$, $\mathrm{F}_{\mathrm{IS}}$ y $\mathrm{F}_{\mathrm{IT}}$ lo que indica un exceso no significativo de individuos heterocigotos $(p>0,05)$. Valores similares de He y de exceso de heterocigotos se presentan en las razas Criollo Araucano (Paz et al. 2014, p. 330), Sangsari (Kasiriyan et al. 2010, p. 33), Afshari y Merhaban (Javanmard et al. 2011, p. 160), Kordi y Arabic (Ghaderi et al. 2010, p.8022). Una de las razones que explicaría el exceso de heterocigotos encontrado puede estar asociado a que dichos animales tienen mayor prolificidad y por tanto se les permite seguir reproduciéndose en el aprisco. De otro lado, en la raza Corriedale Vera et al. (2014, p. 18) presenta un déficit de heterocigotos.

En la muestra poblacional analizada no se encontraron desviaciones significativas del EHW, lo que evidencia la falta de programas se selección y mejoramiento genético direccionado al aumento de la prolificidad en el OPC. Lo anterior también concuerda con la baja variación entre rebaños encontrada (1\%) y con el bajo valor de $\mathrm{F}_{\mathrm{ST}}$ estimado, mostrando la ausencia de una estructura poblacional marcada y la presencia de flujo de genes entre los rebaños.

\section{CONCLUSIONES}

Los loci Fec $X^{\mathrm{R}}$ y FecG $\mathrm{F}^{\mathrm{H}}$ fueron monomórficos, solo se encontró el genotipo silvestre tal vez en razón a efectos de deriva genética, a que los polimorfismos están fijos en las razas en las cuales fueron reportados, a que estas razas no hicieron parte en la formación del OPC o a la falta de planes de manejo reproductivo y de mejoramiento genético en la raza.

El locus FecG ${ }^{\mathrm{I}}$ del gen GDF9 fue polimórfico con mayor frecuencia del genotipo y alelo silvestre, este valor fue similar el reportando en diferentes razas en todo el mundo. No se encontró el genotipo homocigoto $\mathrm{FecG}^{\mathrm{I}} / \mathrm{FecG}^{\mathrm{I}}$, la ausencia de este genotipo puede estar 
asociado a que algunos autores reportan a que este produce ovejas estériles. Se evidenció un exceso de heterocigotos no significativo. No se encontró una estructura poblacional marcada, indicando que las fincas de OPC se comportan como una sola gran población con flujo de genes entre ellas.

\section{AGRADECIMIENTOS}

Al grupo de investigación en reproducción y mejoramiento genético animal de la Universidad de Sucre por la financiación de la investigación y a los productores de OPC del departamento de Córdoba que permitieron la colecta de las muestras.

\section{BIBLIOGRAFÍA}

Alabart J, Lahoz B, Calvo J, Jurado J, Fantova E, Equipo Técnico De Upra- Grupo Pastores \& Folch J, 2016, 'Estudios realizados y situación actual de la variante génica prolífica ROA (FecX) de la raza ovina Rasa Aragonesa', Archivos de Zootecnia, vol. 65, no. 251, pp. 449-52.

Barzegari A, Atashpaz S, Ghabili K, Nemati Z, Rustaei M \& Azarbaijani R, 2010, 'Polymorphisms in GDF9 and BMP15 associated with fertility and ovulation rate in Moghani and Ghezel sheep in Iran', Reproduction in Domestic Animals, vol. 45, no. 4, pp. 666-9.

Buratovich O, 2010, 'Eficiencia reproductiva en ovinos: factores que la afectan. Parte 1: La alimentación', Carpeta técnica ganadera, vol. 34, pp. 1-4.

Carrillo-Gonzales, D \& Hernández, D. 2016, 'Caracterización seminal de individuos ovinos criollos colombianos de pelo en el departamento de Sucre', Revista Colombiana de Ciencia Animal, vol. 8, no. 2, pp. 197-03.

Castellanos J, Rodríguez J, Toro W \& Luengas C, 2010, Agenda prospectiva de investigación y desarrollo tecnológico para la cadena productiva cárnica ovino-caprina en Colombia, Ministerio de Agricultura y Desarrollo Rural, Colombia, visto 30 Noviembre 2017, < https://sioc.minagricultura.gov.co/OvinoCaprina/ Documentos/004\%20-\%20Documentos\%20Competitividad\%20 Cadena/Agenda\%20Prospectiva\%20de\%2OInvestigacion.pdf>.

Cuellar-Gamboa G, Jimenez-Robayo L, Grajels-Lombana H, MoralesMendoza L, Leal-Gutierrez J \& Sanchez-Isaza C, 2015, 'Factores que influencian la prolificidad en ovinos del centro agropecuario marengo, Colombia', Actas Iberoamericanas de Conservación Animal, vol. 6, pp. 460-5.

Davis GH, 2005, 'Major genes affecting ovulation rate in sheep', Genetic Selection and Evolution, vol. 37, Suppl. 1, pp. S1 1-23.

Ghaderi A, Nasari M, Mirzadeh K, Fayazi J \& Sadr A, 2010, 'Identification of the GDF9 mutation in two sheep breeds by using polymerase chain reaction-restriction fragment length polymorphism (PCR-RFLP) technique', African Journal of Biotechnology, vol. 9, no. 47, pp. 8020-22.

Ghaffari M, Nejati-Javaremi A \& Rahimi-Mianji, G, 2009, 'Lack of polymorphism in the oocyte derived growth factor (GDF9) gene in the Shal breed of sheep', South African Journal Animal Science, vol. 39, no. 4, pp. 355-60.

Hanrahan P, Gregan, S, Mulsant P, Mullen M, Davis G, Powell R \& GallowayS, 2004, 'Mutations in the genes for oocytederived growth factors GDF9 and BMP15 are associated with both increased ovulation rate and sterility in Cambridge and Belclare sheep (Ovis aries)', Biology and Reproduction, vol. 70, no. 4, pp. 900-9.

Javanmard A, Azadzadeh N \& Esmailizadeh A, 2011 , 'Mutations in bone morphogenetic protein 15 and Growth Differentiation Factor 9 genes are associated with increased litter size in fat-tailed sheep breeds', Veterinary Research Communications, vol. 35, no. 3, pp. 157-67.
Kasiriyan M, Hafezian S \& Hassani N, 2010, 'Genetic polymorphism BMP15 and GDF9 genes in Sangsari sheep of Iran', International Journal of Genetics and Molecular Biology, vol. 3, no. 1, pp. 31-4.

Kim K, Yeo S \& Choi B, 2002, 'Genetic diversity of north-east Asian cattle based on microsatellite data', Animal Genetics, vol. 33, no. 3, pp. 201-4.

Lahoz B, Alabart J, Jurado J, Calvo J, Martínez-Royo A, Fantova E \& Folch J, 2014, 'Effect of the FecXR polymorphism in the bone morphogenetic protein 15 gene on natural or equine chorionic gonadotropin-induced ovulation rate and litter size in Rasa Aragonesa ewes and implications for on-farm application', Journal Animal Science, vol. 89, no. 11, pp. 3522-30.

Liandris A, Kominakisb A, Andreadoua M, Kapeoldassia K, Chadioa S, Tsiligianni T, Gazoulid M \& Ikonomopoulosa I, 2012, 'Associations between single nucleotide polymorphisms of GDF9 and BMP15 genes and litter size in two dairy sheep breeds of Greece', Small Ruminant Research, vol. 107, no. 1, pp. 16-21.

Liao W, Moore K \& Shimasaki S, 2004, 'Functional and Molecular Characterization of Naturally Occurring Mutations in the Oocytesecreted Factors Bone Morphogenetic Protein-15 and Growth and Differentiation Factor-9', The Journal of Biological Chemistry, vol. 279, no. 17, pp. 17391-6.

Luna C \& Alonso R, 2014, 'Genes con efecto mayor sobre la fertilidad de ovejas. Revisión', Revista Mexicana de Ciencias Pecuarias, vol. 5, no. 1, pp. 107-30.

Martínez-Royo A, Jurado J, Smulders P, Marti I, Alabart A, Roche I, Fantova I, Bodin L, Mulsant P, Serrano M, Folch J, \& Calvo J, 2008, 'A deletion in the bone morphogenetic protein 15 gene causes sterility and increased prolificacy in rasa aragonesa sheep', Animal Genetics, vol. 39, no. 3, pp. 1-4.

Monteagudo L, Ponz R, Tejedor M, Lavina A \& Sierra I, 2009, 'A 17 bp deletion in the Bone Morphogenetic Protein 15 (BMP15) gene is associated to increased prolificacy in the Rasa Aragonesa sheep breed', Animal Reproduction Science, vol. 1 10, no, 1-2, pp. 139-46.

Moradband F, Rahimi G \& Gholizadeh M, 2011, 'Association of polymorphisms in fecundity genes of GDF9, BMP15 and BMP15-1B with litter size in Iranian Baluchi sheep', Asian-Australasian Journal Animal Science, vol. 24, no. 9, pp. $1179-83$.

Ocampo JR, 2014, 'Caracterización genética de ovinos en Colombia por medio de marcadores microsatélites', Tesis de Maestría, Universidad de Antioquia, Medellín, Colombia.

Ocampo RJ, Martínez RA, Rocha JF \& Cardona H, 2017, 'Genetic characterization of Colombian indigenous sheep', Revista Colombiana de Ciencias Pecuarias, vol. 30, no. 2, pp. 116-25.

Paz E, Quiñones J, Bravo S, Rodero E, González A \& Sepúlveda N, 2014. 'Identificación de los polimorfismos G1 y G8 del gen GDF9 en ovinos criollos Araucanos'. Archivos de Medicina Veterinaria, vol. 46, no. 2, pp. 327-31.

Peakall R \& Smouse P, 2012, 'GENALEX 6.5: genetic analysis in Excel. Population genetic software for teaching and research-an update', Bioinformatics, vol. 28, no. 19, pp. 2537-39.

Vivas N, 2013, 'Diversidad genética de ovinos criollos colombianos', Tesis de Maestría, Universidad Nacional de Colombia, Sede Palmira, Colombia.

Vacca G, Dhaouadi A, Rekik M, Carcangiu V, Pazzola M \& Dettori $M, 2010$, 'Prolificacy genotypes genotipes at BMPR 1B, BMP15 and GDF9 genes in North African sheep breeds', Small Ruminant Research, vol. 88, no. 1, pp. 67-71.

Vera J, Bravo S, PazE \& Sepúlveda N, 2014, 'Detección de polimorfismos en el gen de efecto mayor GDF9 en rebaños de ovejas corriedale en la región de Aysén, Chile', Chilean Journal of Agricultural and Animal Science, vol. 31, no. 1, pp. 15-20. 